\title{
Posterior fossa extradural haematomas: operate or conserve?
}

\begin{abstract}
Background: Posterior fossa extradural haematomas (PFEDHs) are an uncommon entity, accounting for only 4 to $12.9 \%$ of all extradural haematomas encountered in clinical practice (EDHs). They have a characteristic clinical presentation, which if detected early, can be life saving. Most PFEDHs are operated owing to the imminent propensity of brainstem compression and death. However, a growing number of neurosurgeons, now consider conservative therapy for smaller bleeds. Clear and reliable criteria do not exist, to assist this critical decision-making process. Hence, here with our clinical experience, we attempt to formulate a clinico-radiologic criteria for the management of PFEDHs. When to operate and when to conserve - the jury is still out on this matter.
\end{abstract}

Methods: The clinical and radiological data of patients with PFEDHs admitted to Kasturba Medical College hospital, Manipal between the period of January 2012 and September 2014 were considered to test the efficacy of the criteria proposed. We carried out a retrospective analysis of 12 patients with PFEDH who were admitted during the above said period. A transverse diameter of $4 \mathrm{cms}$ (of the extradural haematoma) was taken as a cut-off for the decision-making process.

Results: Of the 12 cases reviewed only 4 were operated with a single mortality. All the operated cases had bleeds of a transverse diameter of $>4 \mathrm{cms}$ or had a GCS of less than 13 . $46 \%$ of bleeds occurred due to occipital bone fracture resulting in a diploic venous ooze or a sinus bleed.

Conclusion: PFEDHs are relatively less often encountered in clinical practice. The PF is an unfavorable location for a hematoma. Good GCS scores, at the time of presentation, have a favorable prognosis. Our proposed criteria, for the management of PFEDHs, concluded that all bleeds greater than $4 \mathrm{cms}$ in transverse diameter are to be treated surgically while those with less than $4 \mathrm{cms}$ in diameter are to be managed on the basis of GCS.

Keywords: Posterior fossa extradural, Scoring system, Surgery decision, GCS
Volume 3 Issue 5 - 2015

\author{
Sibhi Ganapathy, Rajesh Nair, Vinod Kumar, \\ Lakshman I Kongwad \\ Department of Neurosurgery, Kasturba Hospital, India
}

\author{
Correspondence: Dr. Rajesh Nair, Neurosurgery Registrar \\ Department of Neurosurgery, Kasturba Hospital, Manipal \\ University, Manipal 576104, India, \\ Email rajeshnair39@yahoo.com
}

Received: November 29, 2015 | Published: December 10, 2015

\section{Introduction}

Posterior fossa extradural bleeds are unique, ${ }^{1}$ as they exist in an anatomical space where large venous channels are located in dangerous proximity to the brainstem. ${ }^{2}$ The nature of the anatomy combined with the rapid expansion of bleeds makes PFEDHs a dreaded traumatic entity. The gold standard of therapy, since age-old days, has been surgery. ${ }^{3}$ However, over the last decade, conservative management, for a select group of PFEDHs, has been tried with success. ${ }^{4}$ There are no clear criteria regarding the decision to observe or to operate, in such cases. Wong and associates proposed one of the many criteria that use clinical and radiological data in advising treatment strategies. ${ }^{5}$ Therefore, this study aims to assess the use of a simple clinico-radiological measure to dictate management strategy in patients with PFEDHs.

\section{Materials and methods}

A retrospective analysis of all posterior fossa EDHs presenting to the emergency of a tertiary care university teaching hospital between the period of January 2012 and December 2014. Data such as demographic profiles, mode of injury, Glasgow Coma Scale (GCS) on arrival, Computed tomographic scan of the Brain (CT Brain) on arrival (where the transverse diameter of the hematoma was measured), management strategy and course of stay in the hospital along with outcomes and 3 weekly follow up in the outpatient department were tabulated and analyzed. Bleeds on either side on the tentorium cerebelli were included in this study, however the transverse diameter of only the posterior fossa component was used in the study.

\section{Results}

$32 \%$ of all cases in the trauma triage were neurosurgical admissions, of which $49.3 \%$ were extradural hematomas. Of these, however, only $12(9.5 \%)$ were in the posterior fossa. This is similar to the expected incidence of PFEDHs reported in the literature.

The demographics were mostly males who were involved in road traffic accidents $(45.5 \%)$, involving two-wheelers. Another important cause of trauma was falling from a height exceeding 5 meters (Table 1).

\section{Table I Demographics}

\begin{tabular}{lll}
\hline & Male $(\mathbf{n}=\mathrm{II})$ & Female $(\mathbf{n}=\mathrm{I})$ \\
\hline $\begin{array}{l}\text { Age in Years (Range) } \\
\text { Mode of Injury }\end{array}$ & $4-67$ & 49 \\
RTA, $\mathrm{n}(\%)$ & $5(45.5)$ & 0 \\
Fall from 2Wheeler, $\mathrm{n}(\%)$ & $2(18 . \mathrm{I})$ & $\mathrm{I}(100)$ \\
Fall from Height >5metres, $\mathrm{n}(\%)$ & $3(27.2)$ & 0 \\
Assault, $\mathrm{n}(\%)$ & $\mathrm{I}(9 . \mathrm{I})$ & 0 \\
\hline
\end{tabular}

The measurement of consciousness, clinically, was made using the Glasgow Coma Scale (GCS). GCS at presentation was stratified into 3 tiers, $>13,8-13$ and $<8$. All patients admitted with a low $\operatorname{GCS}(7,8)$, 
the improved post management. Only one patient who was moribund, preoperatively, succumbed to his ailments, forming the lone mortality in this series. (Figure 1) 50\% of all bleeds had a GCS of over 13. This is comparable to studies in Turkey and Argentina [6,7], where the comparable figure was $97 \%$ and $60 \%$ respectively.

Table 2 Manipal Criteria: bleed size versus GCS

\begin{tabular}{lllll}
\hline & $\begin{array}{l}\text { GCS }<8 \\
\text { Conservative }\end{array}$ & \multicolumn{1}{c}{ Surgery } & Conservative Surgery \\
\hline Bleed Size $<4 \mathrm{cms}$ & 0 & 0 & 8 & $\mathrm{I}$ \\
Bleed Size $>4 \mathrm{~cm}$ & 0 & $\mathrm{I}$ & 0 & 2 \\
\hline
\end{tabular}

Due to the anatomical peculiarities of the posterior fossa, most of the EDHs were fracture hematomas (caused by the diploic venous ooze of the fractured bone, $46 \%$ ), and sinus tears with $46 \%$ bleeds. While the former is self-limiting and has a mild clinical course, the latter may require intensive care and surgery (Figure 2). 50\% of bleeds, again, were due to occipital bone fractures, which were comparable to the standard data available, ${ }^{6,8}$ where the incidence was reported as $57 \%$ in Turkey and $85 \%$ in India.

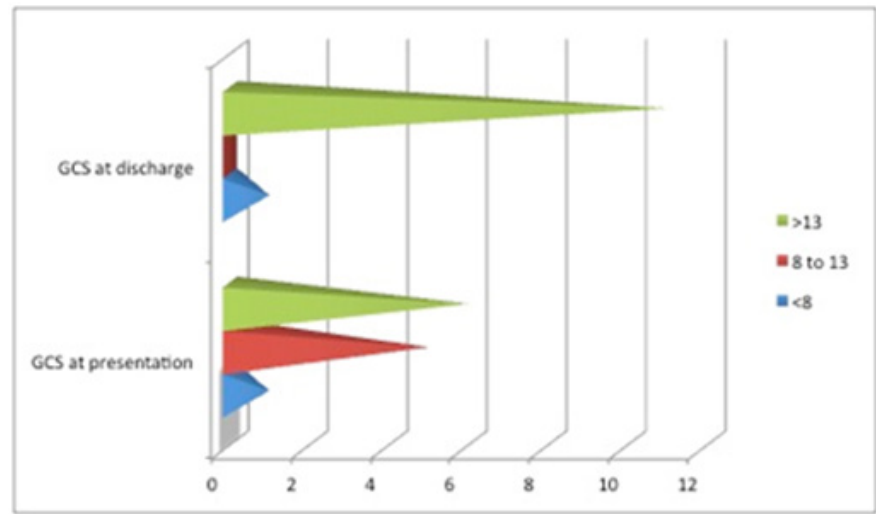

Figure I Comparitive bar graph showing the GCS of the patients at admission and discharge.

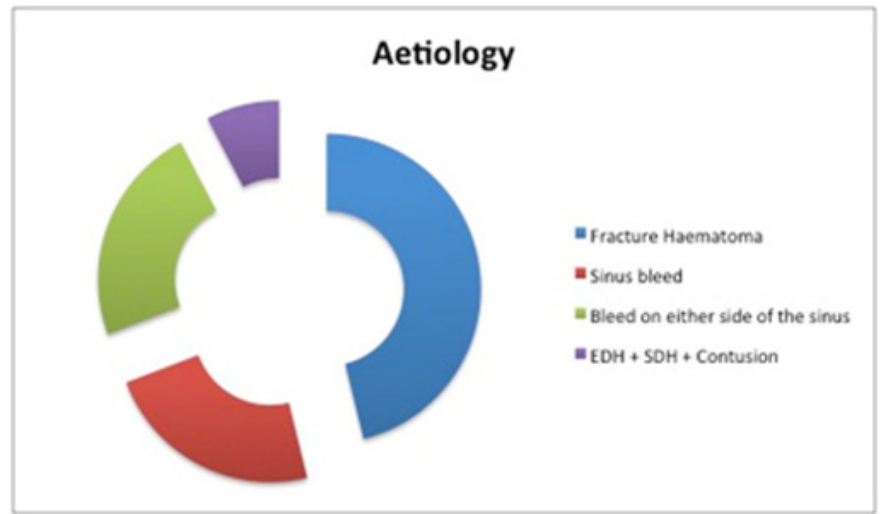

Figure 2 An exploded pie chart showing the various aetiologies encountered in patients with PFEDH.

4 of the 12 cases were managed surgically, with a single mortality, while the others were managed conservatively. Our average hospital stay was 7 days, which is similar to statistics from Germany, ${ }^{9}$ but much shorter compared to data from India (14 days $)^{8}$ At 3 weeks follow-up, post discharge, $46 \%$ reported no adverse effects, while $18 \%$ reported tinnitus and vertigo, commonly seen in PFEDHs (Figure 3 ) this again compared favorably in comparison to studies were done in India $(40 \%)$ and Turkey $(28.5 \%) .6,8$

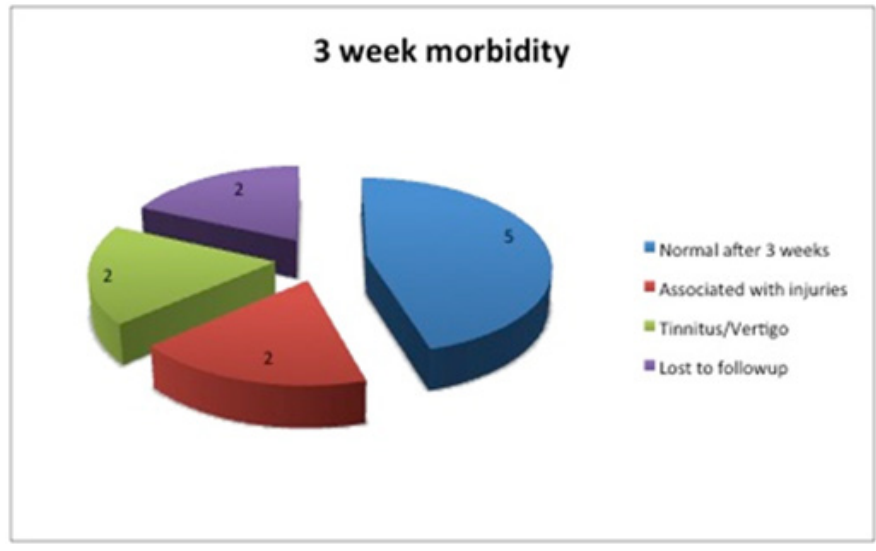

Figure $3 \mathrm{~A}$ pie chart showing the third week mortality in the patients.

\section{Formulation of the manipal criteria}

On comparing GCS with the management strategies employed, it was seen that the lower the GCS, the greater the propensity for surgery (Figure 4) Another comparison of management strategy was the bleed size. This revealed that patients with bleeds larger than $4 \mathrm{~cm}$ in horizontal diameter were proponents for surgical intervention (Figure $5)$.

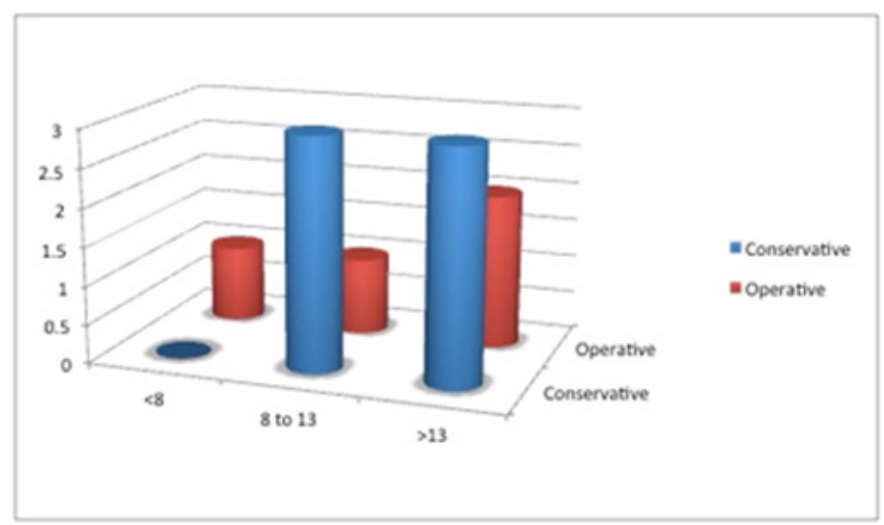

Figure $4 \mathrm{~A}$ comparitive bar graph showing the GCS versus management strategy rendered.

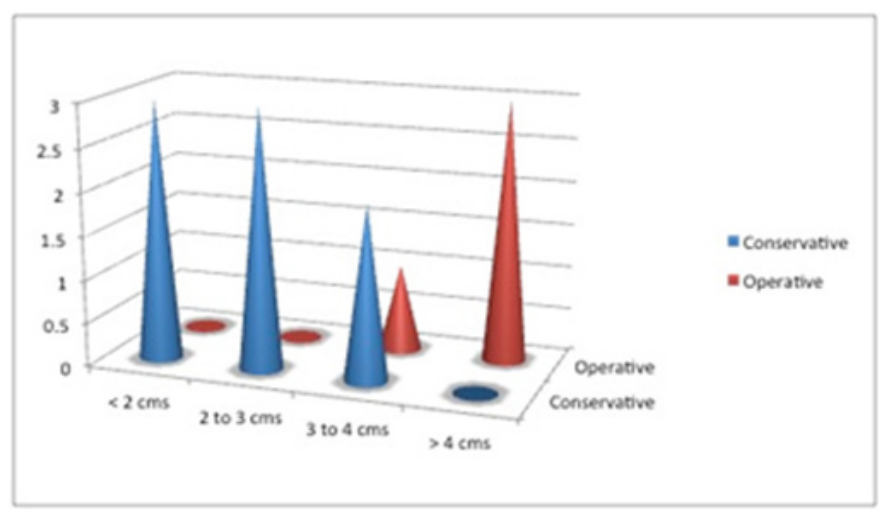

Figure $5 \mathrm{~A}$ comparative bar graph showing the comparision between the posterior fossa bleed size versus management strategy.

This led to the formulation of our criteria for PFEDH management, as shown below (Table 2). All bleeds greater than $4 \mathrm{cms}$ in horizontal diameter were taken up for surgery (Figure 6). For bleeds smaller than $4 \mathrm{cms}$, GCS was employed as the deciding criterion, where patient 
with low GCS despite small bleeds were taken up for decompression and hematoma evacuation, while better GCS patients were observed (Figure 7).

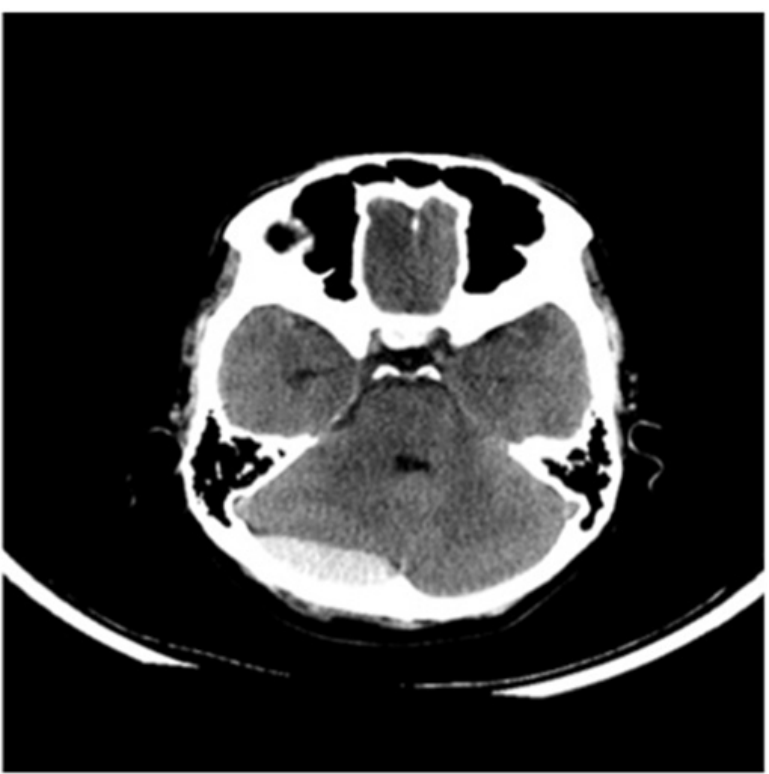

Figure 6 Large posterior fossa extradural bleed,Operative group.

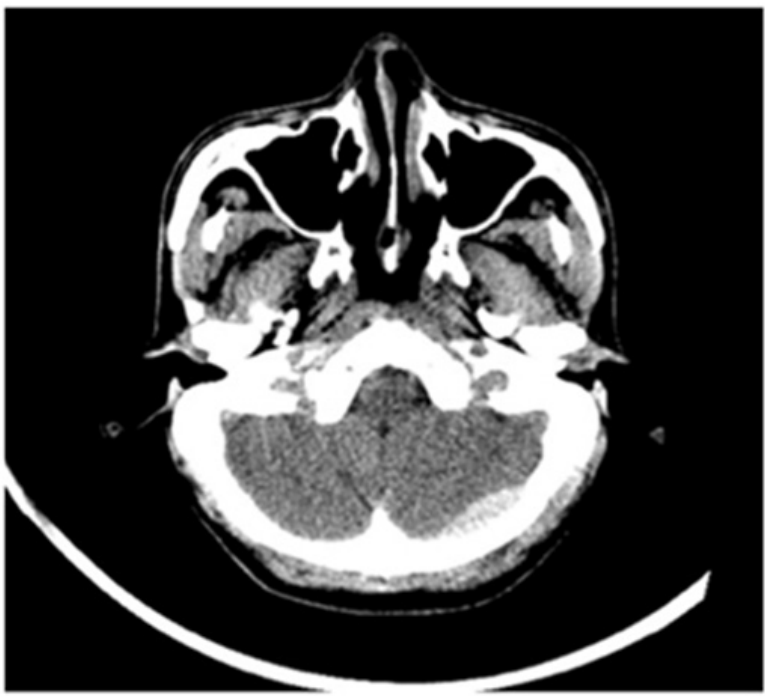

Figure 7 Small posterior fossa extradural bleed, Conservative group.

\section{Conservative management}

This implied close observation of the GCS, Pupils and vitals of the patient in ICU. Neurological deterioration was defined as a decrease in GCS of 2 points from the previous observation reading. The onset of new focal neurological deficits, including brainstem signs, were also considered as deterioration. All neurological deterioration was imaged immediately. 2 patients had significant GCS decrease requiring surgery despite the bleed size not being significant to warrant surgery.

\section{Surgical management}

The standard posterior fossa midline sub occipital craniotomy was performed with hematoma evacuation. In some cases, a V-shaped Dural opening and posterior fossa decompression were done to reduce pressure on the brainstem. No post operative CSF leaks were seen.

\section{Conclusion}

PFEDHS are rare, but deadly, but prompt and appropriate management can yield excellent results. The search for the elusive protocol that may guide the management strategy has led us here. We believe that all bleeds greater than $4 \mathrm{cms}$ in transverse diameter must be operated at the earliest. For those bleeds smaller than $4 \mathrm{cms}$, a watch and wait approach may be employed with close observation of the neurological status of the patient if the GCS is favorable.

It is important to state that management protocols are dynamic and require constant re-evaluation to react appropriately to a changing situation in order to obtain the best results for the patient.

\section{Acknowledgements}

The results of this pilot study, was presented as a poster in the IGCLA 2015 on 21st August 2015 and was awarded the first prize in its category.

\section{Conflicts of interest}

None.

\section{References}

1. Campbell E, Whitefield RD, Greenwood R. Extradural haematomas of the posterior fossa. Ann Surg. 1953;138(4):509-512.

2. Gupta PK, Mahapatra AK, Lad SD. Posterior fossa extradural haematomas. Indian J Pediatr. 2002;69(6):489-494.

3. Hooper RS. Extradural hemorrhages of the posterior fossa. Br J Surg. 1954;42(171):19-26.

4. Bhau KS1, Bhau SS, Dhar S, et al. Traumatic extradural hematoma role of non-surgical management and reasons for conversion. Indian $J$ Surg. 2010;72(2):124-129.

5. Wong CW. The CT criteria for conservative treatment-but under close clinical observation - of posterior fossa epidural hematoma. Acta Neurochir (wien). 1994;126(2-4):124-127.

6. Dirim BV, Orük C, Erdoğan N, et al. Traumatic posterior fossa bleeds. Diagn Interv Radiol. 2005;11(1):14-18.

7. Ahmad FU, Pandey P, Mahapatra AK. Posterior fossa extradural in children. Pediatr Neurosurg. 2005;41(1):49-51.

8. Oliveira MA, Araujo JF, Balbo RJ. Extradural Haematomas of the posterior fossa. Report of 7 cases. Arq Neuropsiquiatr. 1993;51(2):243246. [Article in Portuguese].

9. Rivano C, Altomonte M, Capuzza T, et al. Traumatic posterior fossa Hematomas. A report of 22 new cases surgically treated and a review of the literature. Zentralbl Neurochir. 1991;52(2):77-82. 